\title{
Male mating effort in a polygynous ungulate
}

\author{
Fanie Pelletier • John T. Hogg • Marco Festa-Bianchet
}

Published online: 8 August 2006

(C) Springer-Verlag 2006

Unfortunately, in Fig. 1, an incorrect $x$-axis title appeared. Here is the correct figure:

The online version of the original article can be found at: http://dx.doi. org/10.1007/s00265-006-0208-y.

F. Pelletier • M. Festa-Bianchet

Département de Biologie, Université de Sherbrooke,

2500 Boulevard de 1'Université,

Sherbrooke, Quebec J1K 2R1, Canada

M. Festa-Bianchet

e-mail: M.Festa@Usherbrooke.ca

\section{F. Pelletier $(\bowtie)$}

Division of Biology, Faculty of Natural Sciences,

Imperial College London,

Silwood Park,

Ascot, Berkshire SL5 7PY, UK

e-mail: fanie.pelletier@imperial.ac.uk

\section{J. T. Hogg}

Montana Conservation Science Institute, Limited,

5200 Upper Miller Creek Road,

Missoula, MT 59803, USA

e-mail: jthogg@montana.com
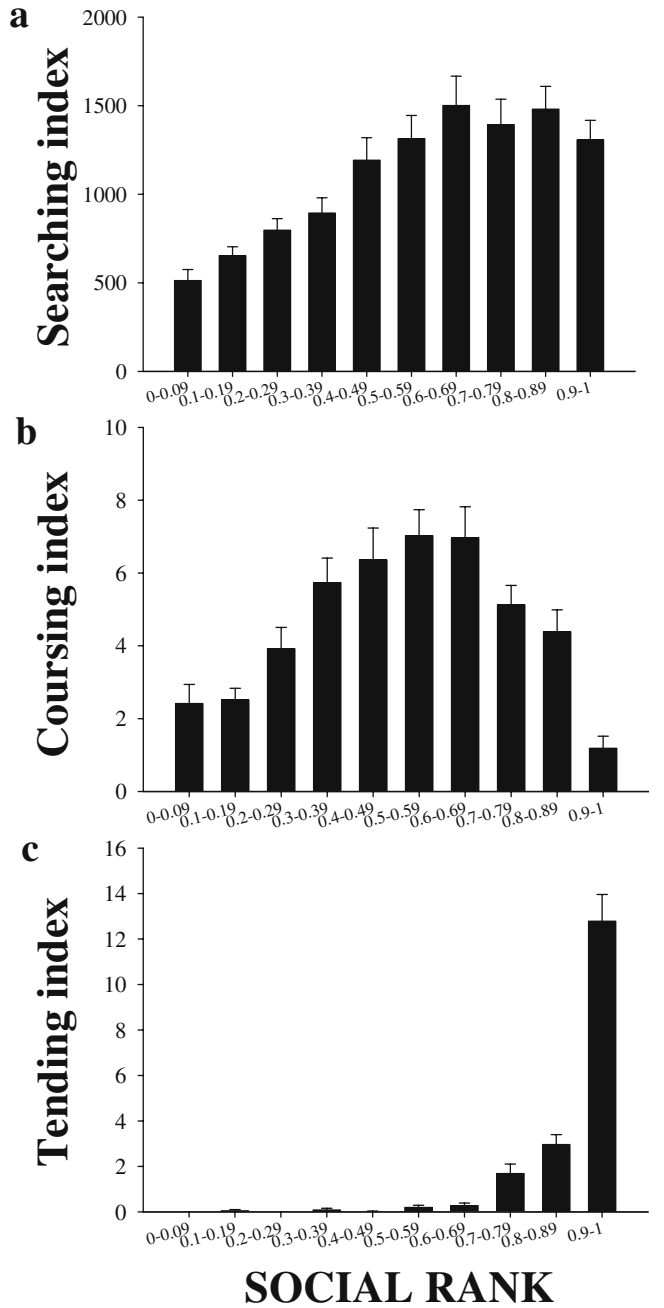

Fig. 1 Rank-specific male mating behavior $( \pm \mathrm{SE})$ for bighorn rams during 14 breeding seasons (1989-2002) at Sheep River, Alberta. a Searching index (in meters per day), b coursing index, and $\mathbf{c}$ tending index 